\title{
Structural Properties of Some Alkali Metal Hydrides at High Temperature and Pressure
}

\author{
V. Thakre AND S. Singh* \\ Department of Physics, Barkatullah University, Bhopal-462026, India \\ (Received January 21, 2014; in final form April 10, 2017)
}

\begin{abstract}
In this paper we have developed a realistic interaction potential model to study the high pressure phase transition of ionic alkali-metal compounds $\mathrm{KH}$ and $\mathrm{NaH}$, having $B_{1}$ structure at room temperature. We have theoretically investigated phase transition pressures and volume collapses of $\mathrm{KH}$ and $\mathrm{NaH}$ at high temperatures, and found results well suited with available experimental data. The elastic constants and their pressure derivatives are reported first time. The thermodynamical properties of $B_{1}$ phase of $\mathrm{KH}$ and $\mathrm{NaH}$ are also predicted. The inclusion of temperature effect in TBIP makes the present model realistic and suitable for theoretical high-pressure studies.
\end{abstract}

DOI: 10.12693/APhysPolA.133.186

PACS/topics: hydrides, phase transition, three-body interaction, volume collapse, elastic constants, cohesive energy

\section{Introduction}

The compounds belonging to $\mathrm{NaCl}\left(B_{1}\right)$ structure are the simplest structure and most suitable for applying new models and theories for validation. The alkali metal hydrides which also crystallize in $B_{1}$ structure are model systems with simplest anion and cations in the periodic table and as potential hydrogen storage material. They have attracted attention of many researchers [1-9]. The study of effect of temperature and pressure on hydrides is also very important from structural point of view [212]. These hydrides undergo structural phase transition $B_{1}-B_{2}$ at elevated pressure [2-6]. The effect of temperature has also been investigated by many workers [1012]. $B_{1}-B_{2}$ transition can be explained by the Berger mechanism [13] by two ion rhombohedral primitive cells. Watanabe, Tokonami and Moromoto (WTM) [14] explained $B_{1}-B_{2}$ transition by rearrangement of atoms associated with intralayer transitions. These transitions have also been studied by two body potentials and first principle periodic Hartee-Fock theory [15, 16].

Energy dispersive X-ray study also shows that $\mathrm{KH}$, $\mathrm{RbH}$, and $\mathrm{CsH}$ transform from $B_{1}-B_{2}$ structure under high pressure [11, 12]. Ghandehari et al. [9] performed most interesting high-pressure experiments for $\mathrm{CsH}$ using energy-dispersive X-ray diffraction (EDXD) with synchrotron radiation. On the other hand, lots of theoretical studies are available on hydrides. The theoretical methods may be certified by comparing results with measured values. Sun et al. [1] studied $B_{1}-B_{2}$ transition in $\mathrm{NaH}$ using $a b$ initio plane wave pseudo-potential density functional theory method. Martins [6] have calculated equation of the alkali hydrides $\mathrm{LiH}, \mathrm{NaH}$ and $\mathrm{KH}$ as a function of pressure using the pseudo-potential method. Their calculated transition pressures for $B_{1}-B_{2}$ in $\mathrm{NaH}$ and $\mathrm{KH}$

*corresponding author; e-mail: drsadhna100@gmail.com are in good agreement with experiment however $\mathrm{LiH}$ is shown to be stable in $B_{1}$ phase up to $140 \mathrm{GPa}$. Ahuja et al. [2] studied $\mathrm{LiH}, \mathrm{NaH}, \mathrm{KH}$ and $\mathrm{RbH}$ by full potential linear muffin tin orbital (FPLMTO) method and reported $B_{1}-B_{2}$ and $B_{2}-C r b$ type transition. But their predicted phase transition pressure $\left(B_{1}-B_{2}\right)$ for $\mathrm{NaH}$ is higher $(37 \mathrm{GPa})$ than experimental $(28-32 \mathrm{GPa})$ values [3]. One of the reasons for this discrepancy may be ignorance of temperature effects, because the experiments are carried out at room temperature and most of the theoretical studies are done at zero temperature $[2,17]$. This creates a need of theoretical study and approach suitable for hydrides. The successful modelling should be based on experimental inputs for good results.

To remove this discrepancy, in the present paper we have incorporated temperature effects in three-body potential model and this has made our model more realistic. Earlier theoretical studies of phase transition with potential model have been carried out by assuming equilibrium at $0 \mathrm{~K}[2,17]$ instead of room temperature $(300 \mathrm{~K})$. Our potential model consists of the Coulomb energy, threebody interactions, and the energies due to the overlap repulsion extended up to the second neighbour ions. The importance of three-body interaction (TBI) is already established [17-24]. The TBI or charge transfer effects (CTE) owe their origin to the quantum mechanical foundation and so to the phenomenological approach in terms of the transfer or exchange of charge between the overlapping electron shells of the adjacent ionic solids. The need of inclusion of TBI forces was emphasized by many workers for betterment of results $[15,25,26]$. Their studies were based on two-body potentials and they remarked that the results could be improved by including nonrigidity in potential model. Also the results based on two body potentials could not explain the Cauchy violation $\left(C_{12} \neq C_{44}\right)$. The deviation from the Cauchy relation is a measure of the contribution from the noncentral many-body force. We have already employed our TBIP approach to study high-pressure behaviour in some 
$B_{3}-B_{1}$ and $B_{1}-B_{2}$ transitions $[17,27,28]$ successfully. Our present model is able to explain the Cauchy violation $\left(C_{12} \neq C_{44}\right)$ in second order elastic constants. We have studied the phase transition pressure and volume change of $\mathrm{KH}$ and $\mathrm{NaH}$ compounds at high temperature and the second order elastic constants are also calculated. The purpose of this work is to study the structural, elastic and thermophysical properties of $\mathrm{KH}$ and $\mathrm{NaH}$ at high temperature. The present potential model includes the long-range Coulombic, three-body interaction, short-range overlap repulsive interaction operative up to second neighbor ions within Hafemeister and Flygare [29] and Cochran [30] approach.

\section{Potential model and method of computation}

It is well known that pressure causes a compression in the crystal, and consequently it alters the charge distribution of the electron shells. As a result of this, a deformation of the overlapping electron shells of the adjacent ions takes place which leads to an increased chargetransfer $[27,28]$. This interaction becomes more important to consider due to the decrease in inter-ionic spacing of the lattice crystal when pressure gets increased and when anions experience sufficient overlap. Besides, enhance in overlap energy the transferred charge due to the overlap in electron shells, modifies the ionic charge which modifies the Coulomb energy by $\{1+(2 n / z)\}$, where $n$ and $z$ are the number of electrons in outermost shell and ionic charge of the compound. The $f(r)$ is the TBI parameter and is dependent on the nearest neighbor distance $(r)$ as [28]:

$$
f(r)=f_{0} \exp \left(\frac{-r}{\rho}\right) .
$$

The effect of TBI is introduced in the expression of Gibbs free energy, $G=U+P V-T S$, in order to obtain the stability condition for a crystal structure. Here $U$ is the internal energy, which at $T=0 \mathrm{~K}$ is equivalent to the lattice energy, $S$ is the vibrational entropy at absolute temperature $T$. Since the theoretical calculation are done at $T=0 \mathrm{~K}$, hence the Gibbs free energy is equivalent to enthalpy $(H)$. This is not a realistic approach because experiments are carried out at room temperature and not at $T=0 \mathrm{~K}$. This fact causes discrepancy in comparability of theoretical results with experimental data. To obtain better comparability with experimental results we have taken account of the room temperature in pressure induced theoretical calculations. The Gibbs free energies for rock salt $\left(B_{1}\right.$, real) and $\mathrm{CsCl}\left(B_{2}\right.$, hypothetical $)$ structures at room temperature $T=300 \mathrm{~K}$ are given by

$$
\begin{aligned}
& G_{B 1}(r)=U_{B 1}(r)+P V_{B 1}-T S_{1}, \\
& G_{B 2}(r)=U_{B 2}(r)+P V_{B 2}-T S_{2},
\end{aligned}
$$

with $V_{B 1}\left(=2.00 r^{3}\right)$ and $V_{B 2}\left(=1.54 r^{\prime 3}\right)$ as the unit cell volumes, $S_{1}$ and $S_{2}$ are the entropies for $B_{1}$ and $B_{2}$ phases, respectively. In fact, the condition for a transition is that the difference in free energy between two phases approaches to zero

$$
\Delta G=\Delta H-T \Delta S=0 .
$$

The first term in energies (2) and (3) are lattice energies for $B_{1}$ and $B_{2}$ structures and they are expressed as

$$
\begin{gathered}
U_{B 1}=\frac{-\left(\alpha_{M} z^{2} e^{2}\right)}{r}-\frac{12 \alpha_{M} z e^{2}}{r} f(r) \\
+6 b \beta_{i j} \exp \left(\frac{r_{i}+r_{j}-r}{\rho}\right) \\
+\sum_{q=i, j} 6 b \beta_{q q} \exp \left(\frac{2 r_{q}-1.41 r}{\rho}\right), \\
U_{B 2}=-\frac{\alpha_{M^{\prime}} z^{2} e^{2}}{r^{\prime}}-\frac{16 \alpha_{M^{\prime}} z e^{2}}{r^{\prime}} f\left(r^{\prime}\right) \\
+8 b \beta_{i j} \exp \left(\frac{r_{i}+r_{j}-r^{\prime}}{\rho}\right) \\
+\sum_{q=i, j} 3 b \beta_{q q} \exp \left(\frac{2 r_{q}-1.154 r^{\prime}}{\rho}\right) .
\end{gathered}
$$

Here $\alpha_{M}\left(\alpha_{M^{\prime}}\right)$ is the Madelung constant for $B_{1}\left(B_{2}\right)$ structure. $\beta_{i j}$ is the Pauling coefficient, $r_{i}\left(r_{j}\right)$ is the ionic radii of $i(j)$ ions, $S_{1}$ and $S_{2}$ are the entropies, $\rho(b)$ are the range (hardness) parameters.

The first terms in Eqs. (5) and (6) are the long-range Coulomb energy, second terms are three-body interactions corresponding to the nearest neighbor separation $r\left(r^{\prime}\right)$ for $B_{1}\left(B_{2}\right)$ phases, third terms are the energies due to the overlap repulsion represented by Born-Mayer potential for $(i, j)$ ions, and extended up to the second neighboring ions $(i i)$ and $(j j)$ in fourth and fifth terms by using Hafemeister and Flygare (HF) type potential. Now the entropy difference in the last term of Eqs. (4) can be calculated from the relation $[19,31,32]$ :

$$
S_{1}-S_{2}=\int_{1}^{2}\left(\frac{C_{1}-C_{2}}{T}\right) .
$$

Here, 1 and 2 stand for the $B_{1}$ and $B_{2}$ phases, $C_{1}$ and $C_{2}$ are the specific heats of two phases at constant pressure and their values can be calculated by the knowledge of the Gruneisen parameter $(\gamma)$ and linear isothermal temperature coefficient $(\beta)$ as

$$
C_{i}=\frac{\left\{\beta V_{i}\left(B_{T}\right)\right\}_{i}}{\gamma_{i}} .
$$

Here, Gruneisen parameter $(\gamma)$ can be calculated by well known formula as follows [33]:

$$
\gamma=\frac{r_{0}}{6} \frac{U^{\prime \prime \prime}\left(r_{0}\right)}{U^{\prime \prime}\left(r_{0}\right)}=\frac{-r_{0}}{6 \rho} .
$$

\subsection{Structural properties}

The $B_{1}$ structure is most stable in these compounds and at high pressure they transform to body centred $B_{2}$ structure. As the stable phase is associated with minimum free energy of the crystal, we have followed the technique of minimization of the Gibbs free energies of real and hypothetical phases. The phase transition occurs when $\Delta G$ approaches zero $(\Delta G \rightarrow 0)$ as shown in Fig. 1.At phase transition pressure $\left(P_{t}\right)$ these compounds undergo a $\left(B_{1} \rightarrow B_{2}\right)$ transition associated with a sudden collapse in volume showing a first-order phase transition. 


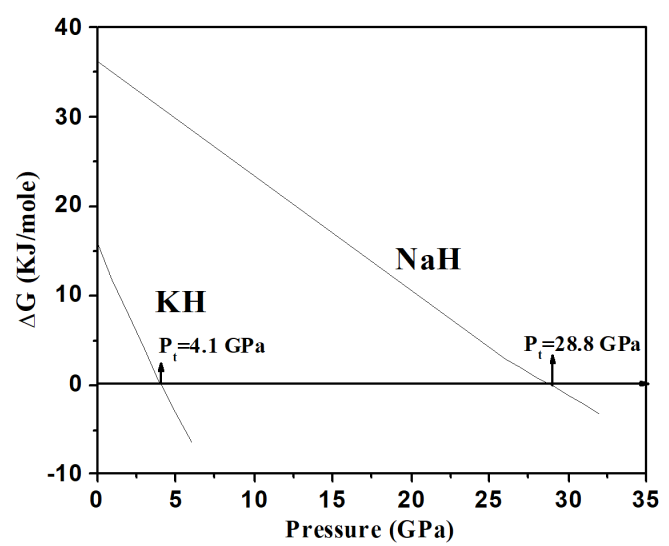

Fig. 1. Variation of DG $(\mathrm{kJ} / \mathrm{mol})$ with pressure for $\mathrm{KH}$ and $\mathrm{NaH}$.

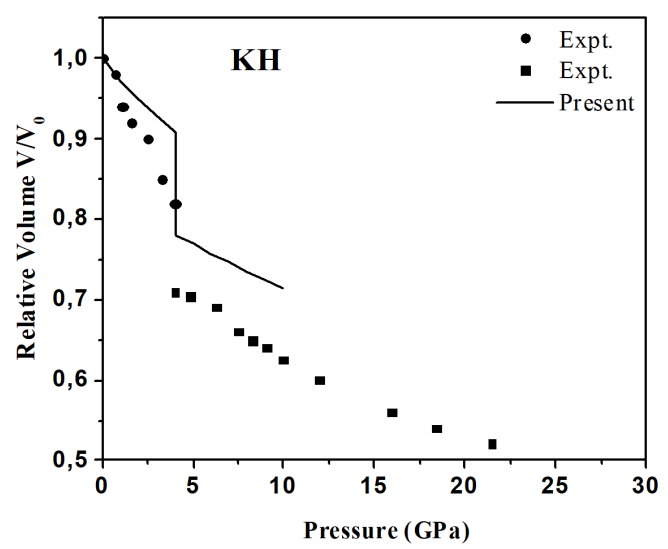

Fig. 2. Variation of relative volumes $V(P) / V(0)$ with pressures in $\mathrm{KH}$. The solid circles represent the experimental data [5] for $B_{1}$ phase and the squares are measured values from Ref. [5] for $B_{2}$ phase.

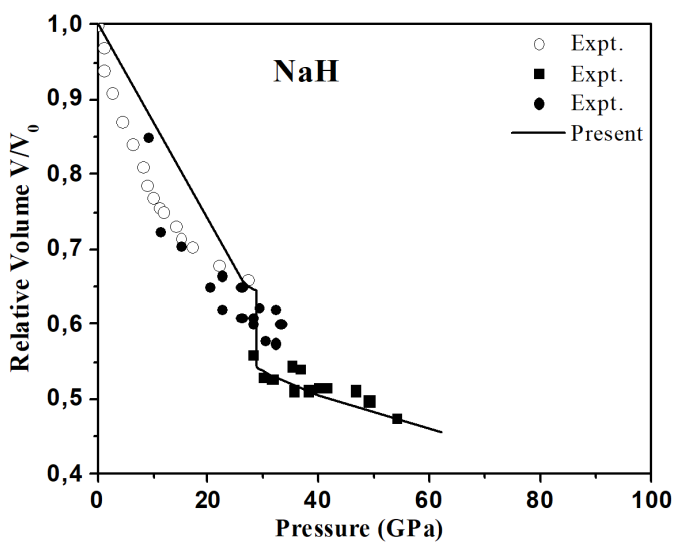

Fig. 3. Variation of relative volumes $V(P) / V(0)$ with pressures in $\mathrm{NaH}$. The solid and open circles represent the experimental data for $B_{1}$ phase from Ref. [3] and Ref. [5], respectively. The squares corresponds to the measured data [3] for $B_{2}$ phase.
Phase transition and volume change of $\mathrm{KH}$ and $\mathrm{NaH}$ at $300 \mathrm{~K}$.

\begin{tabular}{c|c|c|c|c}
\hline \hline Solid & RIPM & Expt. & TBIM (0 K) & Other (0 K) \\
\hline \multicolumn{5}{c}{ Phase transition pressure [GPa] } \\
\hline $\mathrm{KH}$ & 4.10 & $4.00^{a}$ & $5.10^{c}$ & $3.50^{d}$ \\
$\mathrm{NaH}$ & 28.80 & $28.0 \div 32.0^{b}$ & $29.60^{c}$ & \\
\hline \multicolumn{5}{c}{ Volume collapse [\%] } \\
\hline $\mathrm{KH}$ & 13.28 & $13.40^{a}$ & $14.10^{c}$ & $16.40^{e}$ \\
$\mathrm{NaH}$ & 10.26 & $10.00^{b}$ & $9.10^{c}$ & $8.80^{e}$ \\
Refs.: ${ }^{a}[5],{ }^{b}[3],{ }^{c}[17],{ }^{d}[2],{ }^{e}[40]$. &
\end{tabular}

We have also computed the relative volume changes $V(P) / V(0)$ corresponding to the values of $r$ and $r^{\prime}$ at different pressures and plotted them against pressure in Fig. 2 and 3 for $\mathrm{KH}$ and $\mathrm{NaH}$, respectively. It is clear from Table I and Figs. 2 and 3, that our calculated volume collapses from our model for $\mathrm{KH}$ and $\mathrm{NaH}$ are $13.28 \%$ and $10.26 \%$, respectively, which are in better agreement with experimental data $[3,5]$ than others.

\subsection{Elastic properties}

To understand elastic properties of these alkali halides we have calculated second-order elastic constants (SOECs), $\left(C_{11}, C_{12}\right.$ and $\left.C_{44}\right)$ and their pressure derivatives at room temperature $300 \mathrm{~K}$.

The expressions for SOECs $[28,32,34,35]$ are as follows:

$$
\begin{aligned}
& C_{11}=\frac{e^{2}}{4 r_{0}^{4}}\left[-5.112 z(z+12 f(r))+A_{1}+\frac{A_{2}+B_{2}}{2}\right. \\
& \left.\quad+9.3204 z r_{0} f^{\prime}(r)\right], \\
& C_{12}=\frac{e^{2}}{4 r_{0}^{4}}\left[0.226 z(z+12 f(r))-B_{1}+\frac{A_{2}-5 B_{2}}{2}\right. \\
& \left.\quad+9.3204 z r_{0} f^{\prime}(r)\right], \\
& C_{44}=\frac{e^{2}}{4 r_{0}^{4}}\left[2.556 z(z+12 f(r))+B_{1}\right. \\
& \left.\quad+\frac{A_{2}+3 B_{2}}{4}\right] .
\end{aligned}
$$

In Eqs. (10)-(12), first term represents the long-range Coulomb interaction, second term represents the contribution due to TBIP and remaining contribute overlap repulsion expressed in terms of the short-range parameters $\left(A_{1}, B_{1}\right)$ and $\left(A_{2}, B_{2}\right)$ for the nearest neighboring (nn) and next nearest neighboring (nnn) ions.

Using model parameters $(\rho, b, f(r))$, pressure derivatives of SOECs have been computed whose expressions are as follows:

$$
\frac{\mathrm{d} B}{\mathrm{~d} p}=-\frac{1}{3 \Omega}\left[13.980 Z(Z+12 f(r))+C_{1}-3 A_{1}+C_{2}\right.
$$




$$
\begin{aligned}
& \left.-3 A_{2}-167.7648 Z a f^{\prime}(r)+41.9420 z a^{2} f(r)\right] \\
& \frac{\mathrm{d} C s}{\mathrm{~d} p}=-\frac{1}{2 \Omega}\left[23.682 Z(Z+12 f(r))+C_{1}\right. \\
& \quad+\frac{C_{2}+6 A_{2}-10 B_{2}}{4}-50.0752 Z a f^{\prime}(r) \\
& \left.\quad+13.9808 z a^{2} f(r)\right] \\
& \frac{\mathrm{d} C_{44}}{\mathrm{~d} p}=\frac{1}{\Omega}\left[-11.389 Z(Z+12 f(r))+A_{1}-3 B_{2}\right. \\
& \left.\quad+\frac{C_{2}+2 A_{2}-10 B_{2}}{4}+44.6528 z a f^{\prime}(r)\right] \\
& C s=\frac{1}{2}\left(C_{11}-C_{12}\right) \\
& B=\frac{1}{3}\left(C_{11}+2 C_{12}\right) \\
& \Omega=-2.330 Z(Z+12 f(r))+A_{1}+A_{2} \\
& \quad+21.9612 Z a f^{\prime}(r) .
\end{aligned}
$$

The values of $A_{i}, B_{i}$ and $C_{i}(i=1,2)$ have been evaluated from the knowledge of $b, \rho$ and $f(r)$.

\section{Result and discussion}

The present model contains three model parameters $(\rho, b, f(r))$ namely range, hardness and three-body force parameters. The values of these parameters have been evaluated using the value of lattice parameter and the first and second derivatives of the cohesive energy $(U)$ expressed as

$$
\left[\frac{\mathrm{d} U}{\mathrm{~d} r}\right]_{r=r_{0}}=0
$$

and

$$
\left[\frac{\mathrm{d}^{2} U}{\mathrm{~d} r^{2}}\right]_{r=r_{0}}=9 k r_{0} B
$$

Using these model parameters and the minimization technique, phase transition pressures of $\mathrm{KH}$ and $\mathrm{NaH}$ at room temperature have been calculated. The input data of the crystal and calculated model parameters are listed in Table II. Figure 1 shows our present computed phase transition pressures for $B_{1}$ to $B_{2}$ structures in $\mathrm{KH}$ at $4.1 \mathrm{GPa}$ and $\mathrm{NaH}$ at $28.8 \mathrm{GPa}$. The present phase transition pressures are illustrated by arrows in Fig. 1 and their values have been listed in Table I and compared with their experimental and other theoretical results. It is interesting to note from Table I and Fig. 1 that the phase transition pressures $\left(P_{t}\right)$, obtained from our model, are in general in good agreement with experimental data $[3,5]$.

At elevated pressures, the materials undergo structural phase transition associated with a sudden change in the arrangement of the atoms. The atoms are rearranged in new positions leading to a new structure. The compression curves are plotted in Fig. 2 and Fig. 3 for $\mathrm{KH}$

\section{TABLE II}

Input parameters and generated model parameters for $\mathrm{KH}$ and $\mathrm{NaH}$.

\begin{tabular}{c|c|c|c|c|c|c}
\hline \hline \multirow{2}{*}{ Solid } & \multicolumn{3}{|c|}{ Input parameters } & \multicolumn{3}{c}{ Model parameters } \\
\cline { 2 - 7 } & $r_{0}[\AA]$ & $B[\mathrm{GPa}]$ & $\beta[\mu \mathrm{K}]$ & $b\left[\frac{\mathrm{erg}}{10^{12}}\right]$ & $\rho[\AA]$ & $f(r)$ \\
\hline $\mathrm{KH}$ & 2.8590 & $32^{a}$ & $36^{b}$ & 0.0180 & 0.2740 & -0.0072 \\
$\mathrm{NaH}$ & 2.8380 & $19^{a}$ & $64^{b}$ & 0.1260 & 0.3653 & -0.0059 \\
Refs.: & ${ }^{a}[40],{ }^{b}[41]$ & & & &
\end{tabular}

and $\mathrm{NaH}$, respectively. The values of the volume collapses $(-\Delta V(P) / V(0))$ are depicted in Table I. It is clear that during the phase transition from $\mathrm{NaCl}$ to $\mathrm{CsCl}$, the volume discontinuity in pressure volume phase diagram identifies the occurrence of first order phase transition and the trend is the same as found in other studies in $B_{1}$ to $B_{2}[18-24]$ and $B_{3}$ to $B_{1}$ transitions $[28,36]$. The study of elastic behavior under pressure is well known to provide useful information about change in the nature of the covalent and ionic forces induced in the crystal as it is subjected to the phase transformation. The study of SOECs and pressure derivatives of SOECs is important as they provide information about interatomic interactions. We have calculated the variations of the SOECs of the present materials under pressure. Also, we could reproduce the correct sign of the elastic constants $\left(C_{11}-C_{12}\right)$. The study of SOEC's under pressure is important as $C_{11}$ represents elasticity in length and $C_{12}$ and $C_{44}$ are shape related elastic constants. The SOECs and pressure derivatives of $\mathrm{KH}$ and $\mathrm{NaH}$ have been calculated by using Eqs. (10)-(15) and they are given in Table III. The calculated SOECs could not be compared due to lack of experimental data on them. In the case of $\mathrm{NaH}$, the pressure derivative $\mathrm{d} B / \mathrm{d} P$ computed from present model is close to experimental value and other theoretical result.

\section{TABLE III}

Calculated values of elastic constants $[\mathrm{GPa}]$ and pressure derivatives of SOECs and their combinations (in GPa) for $\mathrm{KH}$ and $\mathrm{NaH}$ at $300 \mathrm{~K}$.

\begin{tabular}{c|c|c|c|c|c|c}
\hline \hline Solid & $C_{11}$ & $C_{12}$ & $C_{44}$ & $\mathrm{~d} B / \mathrm{d} P$ & $\mathrm{~d} S / \mathrm{d} P$ & $\mathrm{~d} C_{44} / \mathrm{d} P$ \\
\hline $\mathrm{KH}$ & 45.2000 & 4.6100 & 9.1200 & 6.9480 & 0.5059 & 6.2431 \\
$\mathrm{NaH}$ & 56.5000 & 14.8000 & 25.1000 & 4.2375 & 0.4417 & 1.9810
\end{tabular}

The SOECs of the present compounds have been plotted with pressure in Figs. 4 and 5 for $\mathrm{KH}$ and $\mathrm{NaH}$, respectively. It is clear from Figs. 4 and 5 that in case of $\mathrm{KH}$ and $\mathrm{NaH}, C_{11}$ varies largely under the effect of pressure as compared to the variations in the $C_{12}$ and $C_{44}$. Since the elastic constant $C_{11}$ represents elasticity in length, a longitudinal strain produces a change in $C_{11}$. The elastic constant $C_{12}$ and $C_{44}$ are related to the elasticity in shape, which is a shear constant. A transverse strain causes a change in shape without change in volume. Therefore $C_{12}$ and $C_{44}$ are less sensitive of pressure as compared with $C_{11}$. To the best of our knowl- 


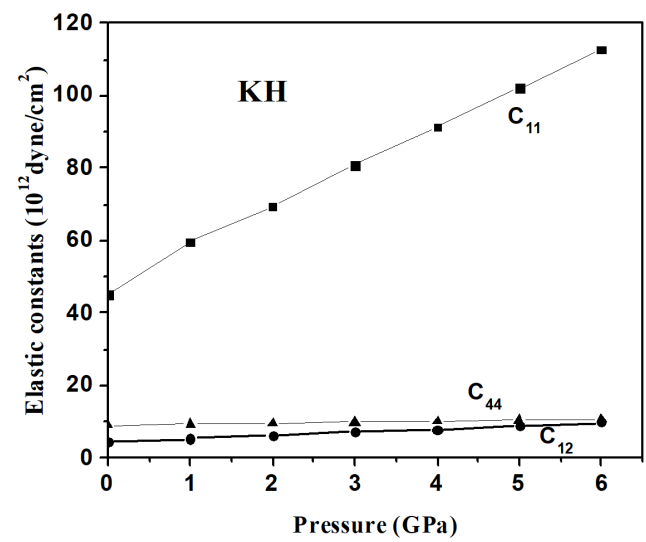

Fig. 4. Variation of elastic constants with pressure for $\mathrm{KH}$.

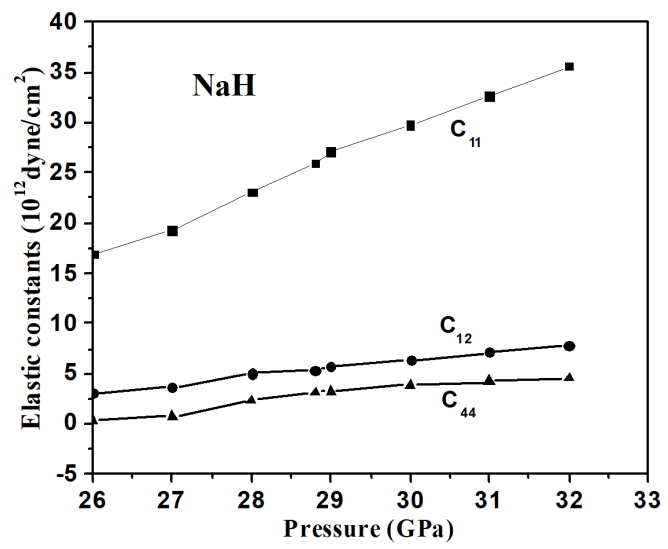

Fig. 5. Variation of elastic constants with pressure for $\mathrm{NaH}$.

edge no experimental and theoretical data of SOECs for these materials are available yet. The deviation from the Cauchy violation $\delta=C_{12}-C_{44}-2 P$ is a measure of the contribution from the non-central many-body force. Our present model is able to explain the Cauchy violation. The calculated values of the Cauchy violation have been given in Table IV for $\mathrm{KH}$ and $\mathrm{NaH}$.

\section{TABLE IV}

Calculated values of anisotropy $A$, shear modulus $G$, the Young modulus $Y$, Cauchy violation $\delta$ and the combinations of elastic constants [GPa] for $\mathrm{KH}$ and $\mathrm{NaH}$ at $300 \mathrm{~K}$.

\begin{tabular}{c|c|c|c|c|c|c|c}
\hline \hline Solid & $A$ & $G$ & $Y \times 10^{4}$ & $\delta$ & $C_{S}$ & $\mathrm{C}_{L}$ & $C_{44} / B$ \\
\hline $\mathrm{KH}$ & 0.4494 & 12.6430 & 13.8425 & -4.51 & 20.3000 & 34.0300 & 0.4800 \\
$\mathrm{NaH}$ & 1.2038 & 23.3039 & 65.8544 & -10.3 & 20.8500 & 60.7500 & 1.3211
\end{tabular}

Vukcevich [37] also stated the high-pressure stability criterion for ionic crystal, the stable phase of a crystal is one in which the shear elastic constant $C_{44}$ is non-zero (for mechanical stability) and which has the lowest potential energy among the mechanically stable lattices. Thus, the stability of NaCl-type structure in terms of elastic constants should satisfy the following conditions: $B=1 / 3\left(C_{11}+2 C_{12}\right)>0, C_{44}>0$ and
$C_{S}=\left(C_{11}-C_{12}\right) / 2>0$. From Table III, our estimated values of $C_{44}$ for $\mathrm{KH}$ and $\mathrm{NaH}$ are positive and hence the above stability criterion [37] are satisfied for ionic crystal. The deviation from the Cauchy relations between SOECs can be expressed accurately in terms of TBIP approach. The values of the combinations of elastic constants $C_{S}=\left(C_{11}-C_{12}\right) / 2, C_{L}=\left(C_{11}+C_{12}+C_{44}\right) / 2$ and $C_{44} / B$ are plotted against pressure and are shown in Figs. 6 and 7 for $\mathrm{KH}$ and $\mathrm{NaH}$, respectively.

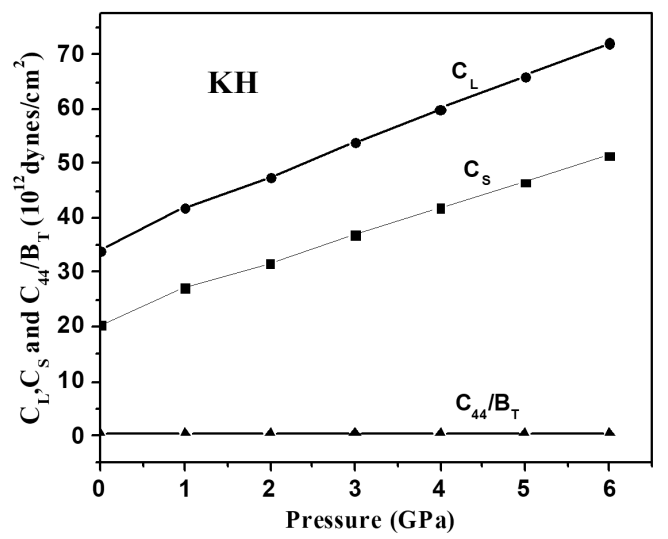

Fig. 6. Variation of combination of elastic constants with pressure for $\mathrm{KH}$.

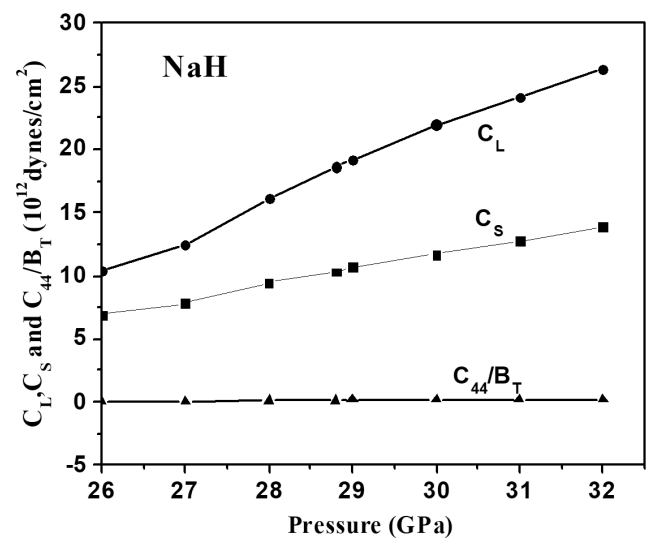

Fig. 7. Variation of combination of elastic constants with pressure for $\mathrm{NaH}$.

We have also plotted the variation of lattice constants (a) with pressure in Figs. 8 and 9. From these figures it is clear that the pressure decreases linearly with the increase in the lattice constants. Also as the size of atom is increased, the phase transition pressure decreases accordingly.

The pressure derivative of the isothermal bulk modulus at a given temperature and pressure $B^{\prime}$ is very important in high-pressure studies. This becomes more important from geophysical viewpoint as it provides information about composition, structure, and texture of solids, as well as the thermal profile of the deep earth. It is also related with the variation in many of the macroproperties of solids with temperature and pressure. So 


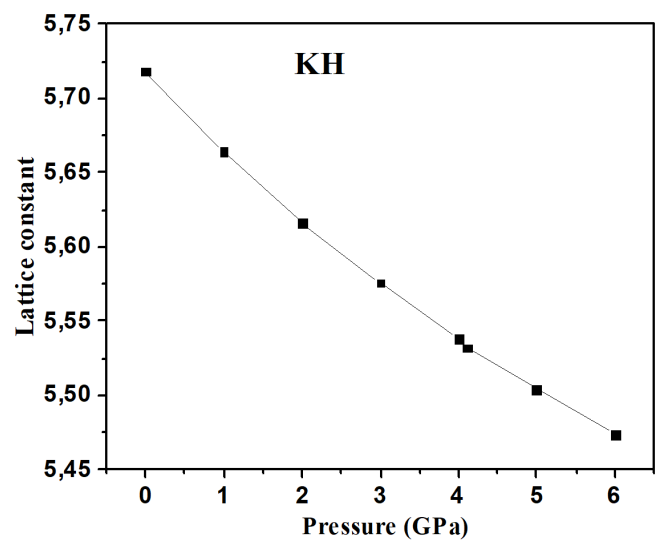

Fig. 8. Variation of lattice constants with pressure for $\mathrm{KH}$.

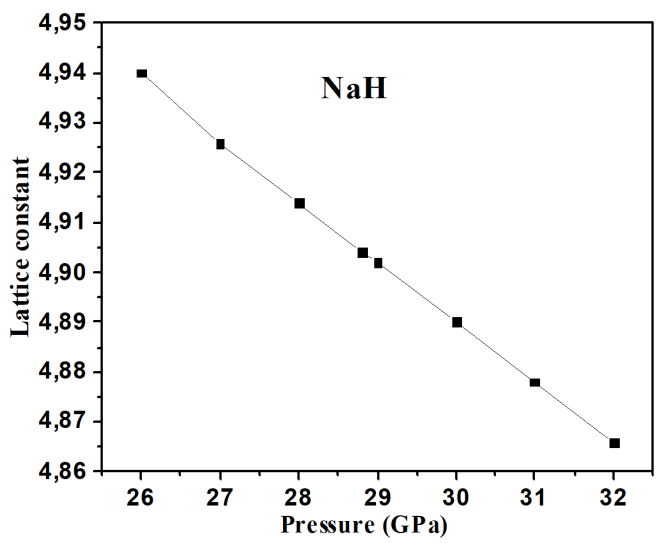

Fig. 9. Variation of lattice constants with pressure for $\mathrm{NaH}$.

we have computed isothermal bulk modulus under pressure $(0$ to $60 \mathrm{GPa})$ with temperatures for $0 \mathrm{~K}$ and $300 \mathrm{~K}$. Figure 10 shows our predicted first pressure derivative of the isothermal bulk modulus $B^{\prime}$ of $\mathrm{NaH}$ with $\mathrm{NaCl}$ - and CsCl-type structures versus pressure at 0 and $300 \mathrm{~K}$. It can be seen that $B^{\prime}$ decreases with the increase of temperature. The results are compared with the experimental [3] and theoretical results [1]. Here we can see that

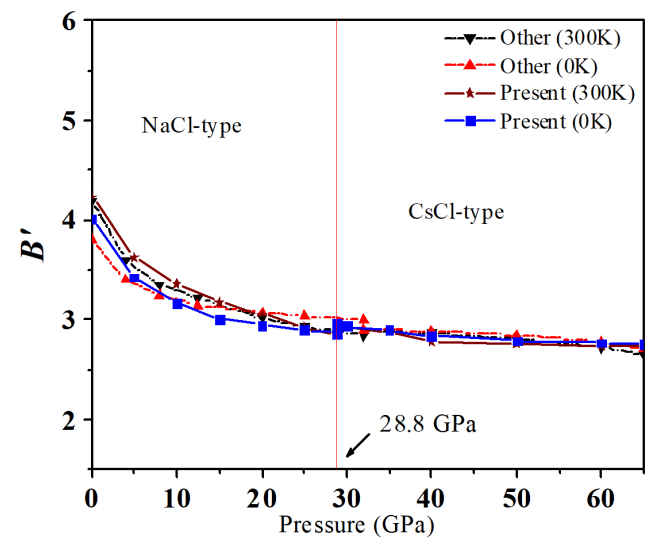

Fig. 10. Variation of first derivative of isothermal bulk modulus with pressure for $\mathrm{NaH}$.
$B^{\prime}$ decreases with the increase of temperature and this decrease is more in the range of $0-15 \mathrm{GPa}$ and this trend is consistent with the results of Sun et al. [1].

It is known that even the cubic crystal which is isotropic structure, has elastic anisotropy as a result of a fourth rank tensor property of elasticity. We have calculated the elastic anisotropic parameter of $\mathrm{KH}$ and $\mathrm{NaH}$ compounds, using the following relation:

$$
A=\frac{2 C_{44}}{C_{11}-C_{12}} .
$$

The calculated values of anisotropic parameter $A$ of $\mathrm{KH}$ and $\mathrm{NaH}$ have been depicted in Table IV. For an isotropic crystal the value of $A$ is 1 . For any values smaller or larger than 1, it indicates the presence of anisotropy. The values of anisotropy in $\mathrm{KH}$ and $\mathrm{NaH}$ show the presence of anisotropy in these compounds.

The isotropic bulk modulus of cubic crystal is given by

$$
B=\frac{C_{11}+2 C_{12}}{3} \text {. }
$$

The shear modulus $G$ can be defined by the following equation:

$$
G=\frac{G_{V}+G_{R}}{2},
$$

where

$$
\begin{aligned}
& G_{V}=\frac{2 C+3 C_{44}}{5}, \\
& G_{R}=15\left(\frac{6}{C}+\frac{9}{C_{44}}\right)^{-1}, \\
& C=\frac{C_{11}-C_{12}}{2} .
\end{aligned}
$$

We have also calculated the Young modulus $Y$, which is related to the bulk modulus $B$ and the shear modulus $G$ by the following equation [38]:

$$
Y=\frac{9 B G}{3 B+G} \text {. }
$$

Our calculated values of bulk modulus $B$, shear modulus $G$, the Young modulus $Y$ of $\mathrm{KH}$ and $\mathrm{NaH}$ compounds at zero pressure are listed in Table IV. These values would not be compared due to unavailability of measured data.

The Poisson ratio of a material influences the speed of propagation and reflection of stress waves. As the Poisson ratio is an important property to know the hardness of compounds, we have calculated the Poisson ratio $(\sigma)$ of present compounds. The expression of $\sigma$ can be given in the following form:

$$
\sigma=\frac{3 B-2 G}{6 B-2 G}
$$

As discussed above the Poisson ratio deals with the way stretching or compressing an object in one direction causes it to compress or stretch in the other direction. The ratio measures the extent of this effect in a particular substance. The Poisson ratio has two limits: it must be greater than -1 , and less than or equal to 0.5 . The calculated values of the Poisson ratio for $\mathrm{KH}$ and $\mathrm{NaH}$ are given in Table $\mathrm{V}$ and they are in above mentioned limit. 


\section{TABLE V}

Elastic wave velocity and the Poisson ratio for $\mathrm{KH}$ and $\mathrm{NaH}$ at $300 \mathrm{~K}$.

\begin{tabular}{c|c|c|c|c}
\hline \hline Solid & $v_{t}$ & $v_{l}$ & $v_{m}$ & $\sigma$ \\
\hline $\mathrm{KH}$ & 6.7928 & 11.3016 & 7.5132 & 0.3487 \\
$\mathrm{NaH}$ & 7.9871 & 12.7915 & 8.7995 & 0.3144
\end{tabular}

The basic material properties, which are of interest in many manufacturing and research applications, can be determined quickly and easily through computations based on sound velocities. Sound velocity can be easily measured using ultrasonic pulse-echo techniques. In addition, to study the thermodynamic properties of these compounds we have calculated the average wave velocity $v_{m}$ on the lines of Guo et al. [39], for calculating the average wave velocity $v_{m}$ the expressions [31] are

$$
v_{m}=\left[\frac{1}{3}\left(\frac{2}{v_{t}^{3}}+\frac{1}{v_{l}^{3}}\right)\right]^{-1 / 3},
$$

where $v_{l}$ and $v_{t}$ are the longitudinal and transverse elastic wave velocities respectively, which are obtained from the Navier equation in the following forms:

$$
\begin{aligned}
& v_{l}=\sqrt{\frac{3 B+4 G}{3 \rho_{D}},} \\
& v_{t}=\sqrt{\frac{G}{\rho_{D}}},
\end{aligned}
$$

where $G$ is the shear modulus, $b$ is the bulk modulus and $\rho_{D}$ is the density. The calculated values of longitudinal and transverse wave velocities are given in Table V. Due to the unavailability of experimental values, we could not compare our results. On the basis of above work, we have concluded our results in the following lines.

\section{Conclusions}

Finally, from the present theoretical investigation we have noticed that:

1. The crystallographic pressure-induced phase transition from $B_{1}$ to $B_{2}$ structure analysis should be done at room temperature $(T=300 \mathrm{~K})$ rather than at $T=0 \mathrm{~K}$.

2. Our calculated phase transition pressures and volume collapses are in general in good agreement with the available experimental and theoretical results.

3. The calculation for second order elastic constants have been performed which have not been performed experimentally yet. They may act as guide to experimentalists.

4. The different combinations of SOECs and thermodynamic properties of alkali halides are predicted by using the present model.
We obtained good comparative results regarding the phase transition pressure and the volume discontinuity in pressure-volume phase diagram with the experimental data. So our present model RIPM is appropriate for the study of phase transition phenomena in present compounds at room temperature. This study may be extended to other compounds of the same structure and to complex structures like $B_{3}$ (zinc blende structure), which is already in progress in our group.

\section{Acknowledgments}

We are thankful to BRNS Mumbai and MAPCOST Bhopal for financial support.

\section{References}

[1] X.W. Sun, Q.F. Chen, X.R. Chen, L.C. Cai, F.Q. Jing, J. Solid State Chem. 184, 427 (2011).

[2] R. Ahuja, O. Eriksson, B. Johansson, Physica B 265, 87 (1999).

[3] S.J. Duclos, Y.K. Vohra, A.L. Ruoff, S. Filipek, B. Baranowski, Phys. Rev. B 36, 7664 (1987).

[4] P. Loubeyre, R. Le Toullec, M. Hanfland, L. Ulivi, F. Datchi, D. Hausermann, Phys. Rev. B 57, 10403 (1998).

[5] H.D. Hochheimer, K. Strossner, W. Honle, B. Baranowsky, S.M. Filipek, Z. Phys. Chem. 143, 139 (1985); J. Less-Common Met. 107, L13 (1985).

[6] J.L. Martins, Phys. Rev. B 41, 7883 (1990).

[7] G.D. Barrera, D. Colognesi, P.C.H. Mitchell, A.J. Ramirez-Cuesta, Chem. Phys. 317, 119 (2005).

[8] N. Geum, G.H. Jeung, R. Cote, A. Dalgarno, J. Chem. Phys. 115, 13 (2001).

[9] K. Ghandehari, H. Luo, A.L. Ruoff, S.S. Trail, F.J. Disalvo, Phys. Rev. Lett. 74, 2264 (1995).

[10] S.B. Roy, P.B. Roy, J. Phys. Condens. Matter 11, 10375 (1999).

[11] X.W. Sun, Y.D. Chu, Z.J. Liu, T. Song, P. Guo, Q.F. Chen, Mater. Chem. Phys. 116, 34 (2009).

[12] M. Kumar, Physica B 217, 143 (1996).

[13] A.R. West, Solid State Chemistry and Its Applications, Wiley, New York 1984.

[14] M. Watanabe, M. Tokonami, N. Moromoto, Acta Crystallogr. A 33, 294 (1977).

[15] C.E. Sims, G.D. Barrera, N.L. Allan, Phys. Rev. B 57, 11164 (1998).

[16] N.L. Allan, G.D. Barrera, J. Purton, C.E. Sims, M.B. Taylor, Phys. Chem. 2, 1099 (2000).

[17] S. Singh, R.K. Singh, High Press. Res. 21, 105 (2001).

[18] P. Bhardwaj, S. Singh, Cent. Eur. J. Chem. 8, 126 (2010).

[19] A. Gour, S. Singh, R.K. Singh, J. Alloys Comp. 468, 438 (2009).

[20] V. Thakre, S. Singh, Opto. Electron. Adv. Mater. 14, 952 (2012).

[21] V. Thakre, S. Singh, J. Phys. Chem. Solids 75, 52 (2014). 
[22] P. Bhardwaj, S Singh, N.K. Gaur, J. Mol. Struct. Theochem. 897, 95 (2009).

[23] P Bhardwaj, S. Singh, Phase Transit. 85, 791 (2012).

[24] P. Bhardwaj, S. Singh, N.K. Gaur, Mater. Res. Bull. 44, 1366 (2009).

[25] S. Froyen, M.L. Cohen, Phys. Rev. B 28, 3258 (1983)

[26] S.B. Zang, M.L. Cohen, Phys. Rev. B 35, 7604 (1987).

[27] R.K. Singh, Phys. Rep. (Netherlands) 85, 259 (1982).

[28] R.K. Singh, S. Singh, Phys. Rev. B 39, 671 (1989); ibid B 45, 1019 (1992); Phase Transit. 15, 127 (1989).

[29] D.W. Hafemeister, W.H. Flygare, J. Chem. Phys. 43, 795 (1992)

[30] W. Cochran, Crit. Rev. Solid State Sci. 2, 1 (1971).

[31] J. Shanker, M. Kumar, Phys. Status Solidi B 179 351 (1993).

[32] J. Shanker, V.C. Jain, J.P. Singh, Phys. Rev. B 22, $1083(1980)$
[33] J. Shanker, W.N. Bhende, Phys. Status Solidi B 136 11 (1986)

[34] P. Bhardwaj, S. Singh, J. Rare Earths 29, 271 (2011).

[35] S. Singh, R.K. Singh, R. Rai, B.P. Singh, J. Phys. Soc. Jpn. 68, 1269 (1999).

[36] S. Singh, M. Sarwan, J. Phys. Chem. Solids 74, 487 (2013); J. Alloys Comp. 550, 150 (2013).

[37] M.R. Vukcevich, Phys. Status Solidi B 54, 435 (1972).

[38] A. Bouhemadou, Comput. Mater. Sci. 43, 1112 (2008).

[39] Y.D. Guo, Z.J. Yag, Q.H. Guo, Z.J. Liu, W. Dai, J. Phys. Condens. Matter 20, 115203 (2008).

[40] C.O. Rodriguez, M.M. Mathfessel, Phys. Rev. B 45, 90 (1992); C.O. Rodriguez, K. Kunc, Solid State Commun. 64, 19 (1987).

[41] Handbook of Physical Quantities, 79th ed., CRC Press, Russia 1997, p. 291. 